\title{
ASO Visual Abstract: Versatility of Retzius-Sparing Prostatectomy - Its Application in Renal Transplant Patients and En Bloc Abdominal-Perineal Resection
}

Chi-Hang Yee, MBBS, FRCS, MD ${ }^{1}$ (i), Sze Jin Adrian Yu, MBChB ${ }^{1}$, Man-Fung Ho, MBChB, FRCS ${ }^{2}$, Kaori Futaba, MBChB, FRCS ${ }^{2}$, Tony Mak, MBChB, MD, FRCS ${ }^{2}$, Jeremy Yuen Chun Teoh, MBBS, FRCS ${ }^{1}$, Peter Ka Fung Chiu, MBChB, FRCS, PhD ${ }^{1}$, Simon Siu-Man $\mathrm{Ng}^{2}$, and Chi-Fai Ng, MBChB, MD ${ }^{1}$

${ }^{1}$ S.H. Ho Urology Centre, Department of Surgery, Prince of Wales Hospital, The Chinese University of Hong Kong, Hong Kong, China; ${ }^{2}$ Division of Colorectal Surgery, Department of Surgery, Prince of Wales Hospital, The Chinese University of Hong Kong, Hong Kong, China

The Retizius-sparing (RS) approach to prostatectomy has several proposed advantages over the conventional retropubic approach. We presented two scenarios where Retzius-sparing robotic-assisted radical prostatectomy (RSRARP) provided additional versatility that was translated into a more desirable clinical outcome (https://doi.org/10. 1245/s10434-021-10804-6). The Retzius-sparing approach is a versatile technique that helps the surgeon to conduct a prostatectomy when special anatomical considerations are at play.
Supplementary Information The online version contains supplementary material available at https://doi.org/10.1245/s10434021-10891-5.

DISCLOSURE All authors have no conflicts of interest to declare.

Publisher's Note Springer Nature remains neutral with regard to jurisdictional claims in published maps and institutional affiliations.

(C) Society of Surgical Oncology 2021

Published Online: 12 October 2021

C.-H. Yee, MBBS, FRCS, MD

e-mail: yeechihang@surgery.cuhk.edu.hk 